\title{
Transformation of sulfur to organic- inorganic hybrids employed by networks and their application for the modulation of refractive indices
}

\author{
AUTHOR(S): \\ Tanaka, Kazuo; Yamane, Honami; Mitamura, Koji; \\ Watase, Seiji; Matsukawa, Kimihiro; Chujo, Yoshiki
}

\section{CITATION:}

Tanaka, Kazuo ...[et al]. Transformation of sulfur to organic-inorganic hybrids employed by networks and their application for the modulation of refractive indices. Journal of Polymer Science Part A: Polymer Chemistry 2014, 52(18): 2588-2595

\section{ISSUE DATE:}

2014-06-13

\section{URL:}

http://hdl.handle.net/2433/200237

\section{RIGHT:}

This is the peer reviewed version of the following article: Tanaka, K., Yamane, H., Mitamura, K., Watase, S., Matsukawa, K. and Chujo, Y. (2014), Transformation of sulfur to organic-inorganic hybrids employed by networks and their application for the modulation of refractive indices. J. Polym. Sci. A Polym. Chem., 52: 2588-2595, which has been published in final form at http://dx.doi.org/10.1002/pola.27274. This article may be used for non-commercial purposes in accordance with Wiley Terms and Conditions for Self-Archiving.; This is not the published version. Please cite only the published version.; この論文は出版社版でありません。引用の際には出版社版をご確認ご利用ください。 


\section{Transformation of Sulfur to Organic-Inorganic Hybrids}

\section{Employed by POSS Networks and Their Application for}

\section{the Modulation of Refractive Indices}

Kazuo Tanaka ${ }^{1}$, Honami Yamane ${ }^{1}$, Koji Mitamura ${ }^{2}$, Seiji Watase ${ }^{2}$, Kimihiro Matsukawa ${ }^{2}$, Yoshiki Chujo $^{1 *}$

${ }^{1}$ Department of Polymer Chemistry, Graduate School of Engineering, Kyoto University, Katsura, Nishikyo-ku, Kyoto 615-8510, Japan

${ }^{2}$ Osaka Municipal Technical Research Institute, 1-6-50, Morinomiya, Joto-ku, Osaka 536-8553, Japan

chujo@chujo.synchem.kyoto-u.ac.jp.

\section{KEY WORDS}

Sulfur, Organic-inorganic hybrid, POSS, Refractive index 


\section{ABSTRACT}

In this manuscript, we present the hybrid materials with sulfur as an inorganic element. Initially, the network polymers with the polysulfide-bridged polyhedral oligomeric silsesquioxane (POSS) were synthesized and mixed with polystyrene (PS) and poly(methyl methacrylate) (PMMA). The resulting polymer blends showed higher thermal degradation temperatures than that of the polymer matrix similarly as typical hybrids. Next, it was found that the sulfur-bridging networks can increase refractive indices of the polymer matrices. Moreover, it was revealed that the loss of Abbe numbers was hardly observed by blending to conventional polymers. 


\section{Introduction}

Organic-inorganic polymer hybrids are materials prepared by mixing organic and inorganic building blocks at the nano or molecular scale. Hybrids have been studied as a platform for developing functional materials because of large potentials to present both advantages intrinsically originated from organic polymers and inorganic materials. As a typical example, by the combination with silicate and conventional polymers, thermally- and mechanically-stable flexible materials can be obtained. ${ }^{1-5}$ Indeed, these stability enhancements to the polymers is beneficial for preparing robust emissive materials. In addition, based on the well-dispersion state in the hybrids at the molecular scale, unique properties derived from the polymer moiety can be observed. ${ }^{6}$ By suppressing the self-quenching and undesired energy transfer between the chromophores incorporated into the hybrids, the bright and pure emissions from each chromophore were detected. ${ }^{6}$ As another instance, Endo et al. have reported the synthesis of sulfur-containing polymers from carbon disulfide. ${ }^{7,8}$ These materials are expected for various application such as the high refractive-index materials and the negative photo-resists. ${ }^{7,8}$ In addition, they also presented the hybrids with organic, sulfur, and zinc system. ${ }^{9}$ Hence, we can expect to obtain not only the preprogrammed functions but also the unexpected characteristics from hybrid materials. So far, despite the diversity of polymers, the most of inorganic elements are classified to the metal oxides, especially silicate. To extend the versatility of organic-inorganic hybrids and to receive further advanced functions, the establishment of the application method with other inorganic elements except for metal oxides on the hybrid formation is necessary.

Sulfur $\left(\mathrm{S}_{8}\right)$ is produced during the purification of petroleum. Even in the resource-poor country like Japan, sulfur is readily available. Most of sulfur is industrially used as a starting material for producing sulfuric acid. Several examples to offer the direct transformation of sulfur to the materials has been achieved. ${ }^{10,11}$ Takata et al. reported a reaction to connect trialkoxysilane structure with oligosulfide bridges. ${ }^{10}$ By using these polysulfide-bridging silane-coupling reagents, various properties of automobile tires can be favorably improved. More recently, the preparation method for the direct transformation of 
sulfur to the plastic was reported. ${ }^{11,12}$ It was demonstrated that the final products can work as a highlyefficient active layer as an electrolyte in the lithium battery. Especially, an introduction of sulfur element into the polymer structure is one of valid strategies for developing high-refractive index plastics ${ }^{13}$ with a high Abbe number, ${ }^{14-19}$ while there are very few examples to demonstrate the optical materials made directly by sulfur $\left(\mathrm{S}_{8}\right)$ as a starting material. Hence, the development of new application manners with sulfur is of significance especially in the industrial fields in terms of the effective utilization of limited resources.

Herein, we present the hybrid materials with sulfur as an inorganic element. The network polymers with the polysulfide linkers were initially synthesized. According to the general classification of the mixture composed of typical polymers such as polystyrene or poly(methyl methacrylate) and inorganic materials, the mixture with high homogeneity are regarded as an organic-inorganic hybrid. Based on this idea, we prepared the polymer blends with the polysulfide-bridging networks and commodity polymers. Similarly as classical silica-based hybrids, the enhancement of thermal stability to the polymer matrices was observed from the measurements of thermal degradation temperature. In addition, it was found that the polysulfide-bridging networks can increase refractive indices of the polymer matrices. Furthermore, it was revealed that the loss of Abbe numbers was hardly observed by blending to conventional polymers. This is the first example, to the best of our knowledge, not only to offer the direct transformation of sulfur to the hybrid materials but also to utilize the sulfur-containing products as an optical material. 


\section{Experimental Section}

General. ${ }^{1} \mathrm{H},{ }^{13} \mathrm{C}$, and ${ }^{29} \mathrm{Si}$ NMR spectra were measured with a JEOL EX-400 (400 MHz for ${ }^{1} \mathrm{H}, 100$ $\mathrm{MHz}$ for ${ }^{13} \mathrm{C}$, and $80 \mathrm{MHz}$ for ${ }^{29} \mathrm{Si}$ ) spectrometer. Coupling constants ( $J$ value) are reported in Hertz. The chemical shifts are expressed in ppm downfield from tetramethylsilane, using residual chloroform $(\delta=$ 7.24 in ${ }^{1} \mathrm{H}$ NMR, $\delta=77.0$ in ${ }^{13} \mathrm{C}$ NMR) or residual tetramethylsilane ( $\delta=0.0 \mathrm{ppm}$ in $\left.{ }^{29} \mathrm{Si} \mathrm{NMR}\right)$ as an internal standard. Scanning electron microscopy (SEM) images were performed using a JEOL JSM-5600 operated at an accelerating voltage of $10 \mathrm{kV}$. Differential scanning calorimetry (DSC) thermograms were carried out on a SII DSC 6220 instrument by using ca. $10 \mathrm{mg}$ of exactly weighed samples at heating rate of $10^{\circ} \mathrm{C} / \mathrm{min}$. The glass transition temperatures $\left(T_{\mathrm{g}}\right)$ were evaluated from the second monitoring curves after annealing at $80^{\circ} \mathrm{C}$ for $5 \mathrm{~h}$, followed by cooling to $30^{\circ} \mathrm{C}$. Thermogravimetric analysis (TGA) was performed on an EXSTAR TG/DTA 6220, Seiko Instrument, Inc., with the heating rate of $10^{\circ} \mathrm{C} / \mathrm{min}$ up to $500{ }^{\circ} \mathrm{C}$ under nitrogen atmosphere. Residual solvents were removed by keeping on the aluminum pan at $120^{\circ} \mathrm{C}$ for 30 min before the TGA measurements. UV-vis absorption spectra were recorded with a SHIMADZU UV-3600 UV-vis-NIR spectrophotometer. Refractive indices $\left(n_{\mathrm{D}}\right)$ were determined with a reflectance spectroscopy film thickness meter (FE-3000, Otsuka Electronics Co., Ltd, Osaka, Japan) at $25^{\circ} \mathrm{C}$. The refractive indices of each wavelength in the visible region were calculated from the fitting of the data to the n-Cauchy approximation equation by entering the film thickness. The $n_{\mathrm{D}}$ values were obtained as the refractive index at $589.3 \mathrm{~nm}$. Abbe numbers $\left(v_{\mathrm{D}}\right)$ were calculated from the refractive indices at $468.1\left(n_{\mathrm{F}}\right), 656.3\left(n_{\mathrm{C}}\right)$, and $589.3 \mathrm{~nm}$ according to equation 4 . The van der Waals volumes of the POSS network and the monomer unit of PMMA and PS were calculated after the modeling using a semi-empirical AM1 method with Spartan '06. The molar refraction of polysulfide was referred to the previous literature. ${ }^{20}$ PS $(\mathrm{DP}=2,000)$ and PMMA $\left(\mathrm{M}_{\mathrm{w}}=700,000-750,000\right)$ were purchased from Wako Pure Chemical Industries, Ltd. (Osaka, Japan) and Nacalai Tesque Inc. (Kyoto, Japan), respectively. The number-average molecular weight $\left(M_{\mathrm{n}}\right)$ and the molecular weight distribution [weight-average molecular weight/number-average molecular weight $\left.\left(M_{\mathrm{w}} / M_{\mathrm{n}}\right)\right]$ values of the POSS network were estimated as an absolute value by size-exclusion chromatography (SEC) with a TOSOH 8020 series [a dual pump system 
(DP-8020), a column oven (CO-8020), and a degasser (SD-8022)] equipped with three consecutive polystyrene gel columns [TOSOH gels: $\alpha-4000, \alpha-3000$, and $\alpha-2500$ ] and a refractive-index (RI-8020) and an ultraviolet detector (UV-8020) at $40{ }^{\circ} \mathrm{C}$. Right-angle light scattering (RALS, light scattering at $90^{\circ}$ ), low-angle light scattering (LALS, light scattering at $7^{\circ}$ ) and refractive index signals were recorded with a Malvern Viscotek 270 Dual Detector. The system was operated at a flow rate of $1.0 \mathrm{~mL} / \mathrm{min}$ with THF as an eluent. Elemental analysis was performed with an AQF-100 (Mitsubishi Chemical Analytical Tech Co., Ltd., Kanagawa, Japan) at the Microanalytical Center of Kyoto University.

Synthesis of the POSS network. Sulfur $\left(\mathrm{S}_{8}, 375 \mathrm{mg}, 1.46 \mathrm{mmol}\right)$ was placed to the bottom of a twonecked flask under argon atmosphere, and distilled THF (30 mL) was added. Then, the small pieces of Na blocks (75 mg, $3.3 \mathrm{mmol}$ ) were added to the suspension, and subsequently the reaction mixture was stirred for $70{ }^{\circ} \mathrm{C}$ for $3 \mathrm{~h}$. After cooling to room temperature, $962 \mathrm{mg}$ of octakis(3-iodopropyl)POSS ${ }^{21}$ (IPOSS, $0.54 \mathrm{mmol}$ ) was added. After stirring at room temperature for $30 \mathrm{~min}$, the reaction was quenched by adding an excess amount of water. The product was extracted with chloroform, and the organic layer was washed with brine. After removing the solvent with a rotary evaporator to the half volume, the POSS network was obtained as the yellow-colored chloroform solution. ${ }^{1} \mathrm{H}$ NMR $\left(\mathrm{CDCl}_{3}\right): \delta=3.22(\mathrm{t}, J=13.8$, 1H), 3.01 (br, 0.4H), 2.09 (br, 0.4H), 1.93 (br, 1H), 0.81 (br, 1.4H) ppm. ${ }^{13} \mathrm{C} \mathrm{NMR}\left(\mathrm{CDCl}_{3}\right): \delta=39.3$, 27.3, 23.3, 21.3, 13.42, 13.37, 11.1, 10.0 ppm. ${ }^{29} \mathrm{Si} \mathrm{NMR}\left(\mathrm{CDCl}_{3}\right): \delta=-67.1,-67.9 \mathrm{ppm}$. Elemental analysis: Calcd. [POSS-(S6) 2.3(I)5.7] C 14.82, H 2.49, S 26.75, I 34.53, [POSS-(S $)_{2.3}(\mathrm{I})_{5.7}$ C C 14.19, H 2.38, S 29.85, I 33.08, Found C 10.33, H 2.24, S 28.38, I 33.31.

Preparation of the blend films. The mixtures $(20 \mathrm{~mL})$ containing $1 \mathrm{~g}$ of polymers (PMMA or PS) and various amounts of the POSS network solution in chloroform were stirred at room temperature for 5 min. Spin coating (1000 rpm) was performed with a MIKASA Spin Coater 1H-D7 (MIKASA Co. Ltd., Tokyo, Japan) by dropping the mixture on the quartz plate $(1 \mathrm{~cm} \times 5 \mathrm{~cm})$. The film samples were dried for 10 
min under ambient condition. The resulting films on the quartz plate were used directly for the refractive index measurements.

Calculation of refractive indices and Abbe numbers of the polymer blends. According to the LorentzLorenz equation, the theoretical values of a refractive index $(n)$ of the polymer matrix (element 1 ) containing the POSS network (element 2) can be described using the molar fractions $(\alpha)$, molar refractions $(R)$, and molar volumes $(V) .^{22}$

$\frac{\mathrm{n}^{2}-1}{\mathrm{n}^{2}+2}=\alpha_{1} \frac{\mathrm{n}_{1}^{2}-1}{\mathrm{n}_{1}^{2}+2}+\alpha_{2} \frac{\mathrm{n}_{2}^{2}-1}{\mathrm{n}_{2}^{2}+2}=\alpha_{1} \frac{R_{1}}{V_{1}}+\alpha_{2} \frac{R_{2}}{V_{2}}$

The degree of packing can be described by the molecular packing coefficient $K_{\mathrm{p}}$ that is defined as

$$
K_{\mathrm{p}}=\frac{V_{\mathrm{VDW}}}{V_{\mathrm{int}}}
$$

where $V_{\text {int }}$ and $V_{\mathrm{VDE}}$ are the intrinsic and van der Waals volumes of the molecules, respectively. According to Vuks equation, equation 1 can be transformed in the equation $3 .^{23}$

$\frac{\mathrm{n}^{2}-1}{\mathrm{n}^{2}+2}=\alpha_{1} \frac{\mathrm{n}_{1}{ }^{2}-1}{\mathrm{n}_{1}^{2}+2}+\alpha_{2} \frac{\mathrm{n}_{2}{ }^{2}-1}{\mathrm{n}_{2}{ }^{2}+2}=\alpha_{1} \frac{K_{\mathrm{p} 1} R_{1}}{V_{\mathrm{VDW}, 1}}+\alpha_{2} \frac{K_{\mathrm{p} 2} R_{2}}{V_{\mathrm{VDW}, 2}}$

The $K_{\mathrm{p}}$ values were determined to be $0.68 .^{24}$ The molar refractions of polymer matrices $R_{1}$ were used according to our previous report. ${ }^{25}$

Abbe numbers $\left(v_{\mathrm{D}}\right)$ were calculated from the refractive indices at $468.1\left(n_{\mathrm{F}}\right), 656.3\left(n_{\mathrm{C}}\right)$, and $589.3 \mathrm{~nm}$ $\left(n_{\mathrm{D}}\right)$ according to equation 4.

$$
v_{D}=\frac{\mathrm{n}_{D}-1}{\mathrm{n}_{F}-\mathrm{n}_{C}}
$$




\section{Results and Discussion}

Refractive indices of the polymeric materials including mixtures and composites can be estimated with the Lorentz-Lorenz equation (equation 1). ${ }^{26}$ Because of high molar refraction of sulfur element, relatively-high refractive indices can be detected. According to equation 4, it is represented that there is the trade-off relationship between a refractive index and an Abbe number: An Abbe number decreases corresponded to the increase of a refractive index of the material. Indeed, by adding ZnS as a filler to poly(vinylpyrrolidone) films, the refractive index increased with drastically decreasing Abbe numbers. ${ }^{27}$ From the series of reports on the development of high-refractive index polymers, it was suggested that the dependency of the refractive index of alkyl sulfide skeletons on the light wavelength intrinsically is small, leading to a high Abbe number.

Without adding the heavy atoms involving sulfur element or aromatic compounds which can efficiently elevate the refractive indices of matrices, it is possible to increase the refractive indices of materials. Nishikubo et al. reported the significant enhancement of refractive indices of polymeric materials by modulating the polymer morphology. ${ }^{28-30}$ They prepared the sulfur element-containing polymers with or without connection of the end of polymer chains. Accordingly, from the series of the refractive index measurements with the polymeric samples with the same sulfur contents, they found that the dendritic polymers showed the highest refractive indices of the samples. Especially, the increase of refractive indices was significantly observed below the glass transition temperatures. ${ }^{31}$ It was summarized that the increase of the fragment density by the end packing should be responsible for the increase of refractive indices. They also proposed that the compact core could be much favorable for observing the larger enhancing effect on the refractive index by the end packing.

As the sulfur containing organic-inorganic hybrids, it was reported that the thiol-modified random silsesquioxanes were applied for the preparation of higher refractive index organic-inorganic hybrids by thiol-ene reaction with allyl compounds. ${ }^{32}$ Polyoctahedral oligomeric silsesquioxanes (POSS, Scheme 1) 
are well-known as molecular building blocks to construct thermally-stable materials as well as to improve the mechanical properties of materials. ${ }^{33-39}$ The rigid POSS structure can suppress the motions of surrounding molecules in media. ${ }^{40-45}$ In addition, the symmetrical structure of the silica cube could contribute to reducing entropy differences in phase transitions. ${ }^{46}$ Thereby, the molecular motion could be suppressed, resulting in the improvements of the thermal stabilities of POSS-containing composites. ${ }^{47}$ The silica cage of POSS has another structural feature. POSS can be regarded as the linker with the compact size. For instance, the POSS-core dendrimers have smaller sizes than those of poly(amidoamine) dendrimer with the same generation possessing the ethylenediamine core. ${ }^{4-50}$ As a result, the guest molecules can be accumulate into the compact spaces by encapsulating into the POSS-core dendrimer. In summary, by using POSS as a cross-linking unit, the polymer chains can be accumulated into the compact spaces, resulting in the increase of local density. In addition, the molecular motions of polymer chains should be efficiently suppressed. These effects are favorable for increasing the refractive indices of polymers. ${ }^{28-30}$

\section{Scheme 1}

Synthesis of the POSS network was carried out by employing the substitution reaction from the iodine groups of I-POSS $^{14}$ with sodium polysulfides according to the Takata's reports ${ }^{10}$ (Scheme 1). In tetrahydrofuran, sulfur was treated with sodium metal, and then I-POSS was added. As a preliminary experiment, the length of the oligosulfide chain was estimated by the ${ }^{1} \mathrm{H}$ NMR spectroscopic analysis of the products obtained by the reaction of the sodium salt with benzyl bromide as reported by Takata et al. (Figure S2). ${ }^{10}$ Accordingly, it is suggested that the average length was determined as five sulfur atoms. After stirring the mixture, the products were extracted with chloroform. By condensing with a rotary evaporation, the pale yellow colored sample solution was prepared. When the solvent was removed completely, the residue was never dissolved in chloroform again. Therefore, the solution state was kept for the storage. 
The characterization of the product was executed. From the integration ratios of the ${ }^{1} \mathrm{H}$ NMR peaks at the propyl moiety in POSS as shown in Figure 1a, the introduction ratio of polysulfide chains can be estimated as $29 \%$ of the eight vertices. In the ${ }^{29} \mathrm{Si}$ NMR spectrum, the significant signal was observed at -67 ppm assigned to the T8 structure of silsesquioxane (Figure 1b). This result indicates that the cubic silica cage should be maintained after the reaction with sodium salt. Based on NMR and elemental analysis data, the average number of polysulfide chains in the single POSS unit was evaluated as approximately between 6 and 7 sulfur atoms. This value is similar to the result from the preliminary experiment with the benzyl bromide treatment. From these data, the averaged molecular weight of the single POSS unit was calculated. Finally, the absolute molecular weight was determined with the GPCLALS measurements (Figure 2). The new peak $\left(M_{\mathrm{n}}=1,200, M_{\mathrm{w}} / M_{\mathrm{n}}=1.47\right.$ ) was observed in the higher molecular weight region than that of I-POSS $\left(M_{\mathrm{n}}=300, M_{\mathrm{w}} / M_{\mathrm{n}}=1.08\right)$ in the profile. The degree of polymerization was calculated as 4 of POSS units. From these data, we concluded that the POSS network with the polysulfide linkers can be obtained. The signal peaks from thiol species were hardly obtained from the ${ }^{1} \mathrm{H}$ NMR spectra. It is implied that most of the end thiol groups might be reconnected between the neighbor thiol groups after the reaction quenching. This fact suggests that the crosslinking could occur not only via the direct connection between POSS by the sodium salts of polysulfide but also by the reconnection between the thiol groups after reaction quenching. We confirmed that this preparation method possesses good reproducibility. From the comparison of ${ }^{1} \mathrm{H}$ NMR spectra before and after storage for 1 month under the ambient condition in the chloroform solution, peak changes or appearances were hardly observed. These data indicate that the synthesized POSS network has high stability toward air and light.

Figures 1 and 2 
The polymer blends of the synthesized POSS network with conventional polymers (PMMA and PS) were prepared (Figure 3). Transparent films were provided as clear films from spin coating on the quartz substrate. The surface morphologies of the blend films were examined using SEM. Significant phase separation or aggregation was slightly observed in the blend films under $20 \mathrm{wt} \%$ POSS network with both matrices (Figure 4 and Figure S3). These results suggest that homogeneous dispersions of the POSS network were realized in polymers under $20 \mathrm{wt} \%$. These data mean that the polymer blends satisfy the definition of hybrid materials where the organic and inorganic components are mixed at the nano or molecular scale. In other words, just by simply mixing with the POSS network, the organic-inorganic hybrids can be obtained.

\section{Figures 3 and 4}

Transparency of the blend films was evaluated with UV-vis absorption measurements (Figure S4). Significant light absorption was hardly observed in the visible region. These data indicate that the blend films possess high transparency. In the UV region, small absorption was detected. According to the previous work, the suppression of the light absorption in the UV region is feasible to maintain high level of Abbe numbers. ${ }^{22,51}$ It is suggested that POSS network could satisfy the demands for suppressing loss of Abbe numbers.

Thermal stability of the blend films was monitored by TGA (Table 1). From the PMMA blends, the second degradation attributed to the main chain scission was suppressed by the addition of POSS network (Figure 5). It is likely that the molecular motion of PMMA main-chains was suppressed by the strong hydrophobic interaction between POSS and polymer main-chains, leading to the enhancement of thermal stability of polymer matrices. ${ }^{47}$ These data also support the statement that the POSS network can form a hybrid with polymer matrices. From the PS blend film, a slightly lower degradation temperature was observed. Since the aggregation of benzene rings could intrinsically occur in the PS film, it is implied that 
the polymer blending might reduce the interaction among polymer chains, resulting in the decrease of the $T_{\mathrm{d}}$ value. We also prepared the blend films with the polysulfide containing propyl groups instead of POSS. However, the degradation occurred at the drying step. This fact supports the POSS effect on the improvement of the stability of the materials. From the DSC measurements with the polymer blends, the decreases of $T_{\mathrm{g}} \mathrm{s}$ of the polymer matrices were observed (Table 1). These data also support well dispersion states of the POSS network in the polymer blends.

\section{Table 1 and Figure 5}

Refractive indices of the blend films were measured. The values were determined as an average from the three data sets of at least five points in the same film. The theoretical values were estimated from the below procedure. In the case of amorphous polymers, the $K_{\mathrm{p}}$ values were determined to be $0.68 .^{24} V_{\text {VDwS }}$ were estimated with the computer modeling. The molar refractions of polymer matrices $R_{1}$ were used according to our previous report. ${ }^{25}$ The molar refraction of the POSS network was calculated by using that of sulfur element in polysulfide according to Figure S1. ${ }^{20}$ Theoretical values were represented from the sum of the experimental values of the pure polymer films and the calculated values obtained the above equations. The results are shown in Figure 6a and Table S1. Corresponded to the increases of the amount of the POSS network, the increases of the refractive indices were observed in both polymer matrices. By adding $10 \mathrm{wt} \%$ POSS networks, the PMMA film showed the increase of refractive indices $\left(1.4994, \Delta n_{\mathrm{D}}\right.$ $=0.0063)$. From the PS film with 20 wt\% POSS networks, the increase of the refractive index (1.4994, $\left.\Delta n_{\mathrm{D}}=0.0106\right)$ was observed. This fact clearly indicates that the POSS network should be responsible for increasing the refractive indices of polymer matrices. The higher values were obtained from the experiments than those of the theoretical values (10 wt\% PMMA sample, 1.4942; 20 wt\% PS sample, 1.5744). These data are explained by three issues. First, similarly as the Nishikubo's reports ${ }^{28-31}$, the fragment density of polysulfides could be enhanced by the connection with POSS. Second, because of the strong hydrophobicity of the silica cube, there is a possibility to generate the strong interaction between 
the POSS units and the polymer chains. As a result, the POSS core could induce the assembly of the mainchains of the polymer matrices. Thereby, the local density in the matrices increased. It means the increase of packing coefficients in the Lorentz-Lorenz equation (equation 1), resulting in the increase of refractive indices. Third, the molar refraction of polysulfide might be higher than the calculated value from the refractive index of sulfur. These three factors could cooperatively increase the refractive indices of the polymer matrices. The Abbe numbers were calculated from the refractive index data according to equation 4 (Figure 6b). Interestingly, less significant changes were observed by increasing the amounts of the POSS network in both polymer matrices. The Abbe numbers are crucially influenced by the chemical composition of the materials in the glass materials. ${ }^{51}$ Therefore, it is assumed that by increasing the local density of matrices, only the high-refractive indices might be induced without loss of an Abbe number. In addition, it is also presumed that the intrinsic Abbe number of the POSS network might be in the similar range to those of polymers. Hence, the crucial changes could be detected in this concentration range of the POSS network.

Figure 6 


\section{Conclusions}

According to the classification of the mixtures containing polymers and inorganic materials, the polymer blends with conventional polymers and the sulfur-bridged POSS network are regarded as a hybrid in this study. Our results involve three significant remarks: First, we demonstrate the preparation of the hybrid materials from sulfur based on the polymer blend. The hybrid film composed of polysulfide bonds was obtained. These characteristics could be applicable for the development of stimuli-responsive materials based on a variety of sulfur element-related chemical reactions. Second, by employing POSS, the homogeneous and thermally-stable hybrids can be readily obtained just with polymer blending. It is extremely easy to change the type of polymers. In addition, there is possibility that the hybrid materials might be produced by using substituted-POSS with other inorganic elements. Third, the synthesized network can work as a filler for increasing refractive indices of polymer matrices without critical loss of Abbe number. In particular, the connection with POSS could increase local density, resulting in the increase of refractive indices of the polymer matrices. Thus, it can be demonstrated that our concept presented here is advantageous not only for the facile preparation of hybrid materials except for metal oxide but also for the development of advanced functional materials.

\section{Acknowledgement}

This research was partially supported by the Suzuki Foundation (for K.T.) and a Grant-in-Aid for Scientific Research on Innovative Areas “New Polymeric Materials Based on Element-Blocks (No.2401)” (25102521) of The Ministry of Education, Culture, Sports, Science, and Technology, Japan. 


\section{References}

1. Kajiwara, Y.; Tanaka, K.; Chujo, Y. Polym. J. 2014, 46, 195-199.

2. Tanaka, K.; Jeon, J.-H.; Inafuku, K.; Chujo, Y. Bioorg. Med. Chem. 2012, 20, 915-919.

3. Kajiwara, Y.; Nagai, A.; Chujo, Y. J. Mater. Chem. 2010, 20, 2985-2992.

4. Kajiwara, Y.; Nagai, A.; Chujo, Y. Bull. Chem. Soc. Jpn 2011, 84, 471-481.

5. Kokado, K., Iwamura, T., Chujo, Y. Polym. J. 2008, 40, 402-408.

6. Kajiwara, Y.; Nagai, A.; Tanaka, K.; Chujo, Y. J. Mater. Chem. C 2013, 1, 4437-4444.

7. Hirata, M.; Abe, Y.; Ochiai, B.; Endo, T. J. Polym. Sci., Part A: Polym. Chem. 2010, 48, 4385-4392.

8. Hirata, M.; Ochiai, B.; Endo, T. J. Polym. Sci., Part A: Polym. Chem. 2010, 48, 525-531.

9. Ochiai, B.; Konta, H. Nanoscale Res. Lett. 2013, 8, 373-375.

10. Yamada, N.; Furukawa, M.; Nishi, M.; Takata, T. Chem. Lett. 2002, 31, 454-455.

11. Chung, W. J.; Greicel, J. J.; Kim, E. T.; Yoon, H.; Simmonds, A. G.; Ji, H. J.; Dirlam, P. T.; Glass, R.

S.; Wie, J. J.; Nguyen, N. A.; Guralinick, B. W.; Park, J.; Somogyi, Á.; Theato, P.; Mackay, M. E.; Sung, Y-E.; Char, K.; Pyun, J. Nat. Chemm. 2013, 5, 518-524.

Simmonds, A. G.; Griebel, J. J.; Park, J.; Kim, K. R.; Chung, W. J.; Oleshko, V. P.; Kim, J.; Kim, E. T.;

Glass, R. S.; Soles, C. L.; Sung, Y.-E.; Char, K.; Pyun, J. ACS Macro Lett. 2004, 3, 229-232.

13. Suzuki, Y.; Murakami, K.; Ando, S.; Higashihara, T.; Ueda, M. J. Mater. Chem. 2011, 21, $15727-15731$.

14. Okubo, T.; Kohmoto, S.; Yamamoto, M. J. Mater. Sci. 1999, 34, 337-347.

15. Lü, C.; Cui, Z.; Wang, Y.; Yang, B.; Shen, J. J. Appl. Polym. Sci. 2003, 89, 2426-2430. 
16. Suzuki, Y.; Higashihara, T.; Ando, S.; Ueda, M. Eur. Polym. J. 2010, 46, 34-41.

17. Suzuki, Y.; Higashihara, T.; Ando, S.; Ueda, M. Polym. J. 2009, 41, 860-865.

18. Okutsu, R.; Ando, S.; Ueda, M. Chem. Mater. 2008, 20, 4017-4023.

19. Suzuki, Y.; Higashihara, T.; Ando, S.; Ueda, M. Macromolecules 2012, 45, 3402-3408.

20. Strecker, W.; Spitaler, R. Ber. 1926, 59, 1754-1775.

21. Dittmar, U.; Hendan, B. J.; Flörke, U.; Marsmann, H. C. J. Organomet. Chem. 1995, 489, $185-194$.

22. Groh, W.; Zimmermann, A. Macromolecules 1991, 24, 6660-6663.

23. Vuks, M. F. Opt. Spectrosc. 1964, 68, 441-451.

24. Tanio, N.; Irie, M. Jpn. J. Appl. Phys., Part I 1997, 36, 743-748.

25. Jeon, J.-H.; Tanaka, K.; Chujo, Y. J. Polym. Sci. Part A: Polym. Chem. 2013, 51, 3583-3589.

26. Tanaka, K.; Adachi, S.; Chujo, Y. J. Polym. Sci. Part A: Polym. Chem. 2010, 48, 5712-5717.

27. Zhang, Q.; Goh, E. S. M.; Bauerman, R.; Judeh, Z.; Chan-Park, M. B.; Chen, T.; Xu, R. J. Appl. Polym. Sci. 2013, 129, 1793-1798.

28. Kudo, H.; Inoue, H.; Inagaki, T.; Nishikubo, T. Macromolecules 2009, 42, 1051-1057.

29. Kudo, H.; Inoue, H.; Nishikubo, T.; Anada, T. Polym. J. 2006, 38, 289-297.

30. Kudo, H.; Sato, K.; Nishikubo, T. Macromolecules 2010, 43, 9655-9659.

31. Kudo, H.; Aoki, H.; Nishikubo, T. Chem. Lett. 2008, 37, 282-283.

32. Matsukawa, K.; Fukuda, T.; Watase, S.; Goda, H. J. Photopolym. Sci. Technol. 2010, 23, 115-119.

33. Guo, H.; Meador, M. A. B.; McCorkle, L.; Quade, D. J.; Guo, J.; Hamilton, B.; Cakmak, M.; Sprowl, G. ACS Appl. Mater. Interfaces 2011, 3, 546-552. 
34. Zhang, Q.; He, H.; Xi, K.; Huang, X.; Yu, X.; Jia, X. Macromolecules 2011, 44, 550-557.

35. Dasari, A.; Yu, Z. Z.; Mai, Y. W.; Cai, G. P.; Song, H. H. Polymer 2009, 50,1577-1587.

36. Jeon, J.-H.; Tanaka, K.; Chujo, Y. J. Mater. Chem. A 2014, 2, 624-630.

37. Jeon, J.-H.; Tanaka, K.; Chujo, Y. RSC Adv. 2013, 3, 2422-2427.

38. Tanaka, K.; Inafuku, K.; Naka, K.; Chujo, Y. Org. Biomol. Chem. 2008, 6, 3899-3901.

39. Tanaka, K.; Chujo, Y. J. Mater. Chem. 2012, 22, 1733-1746.

40. Tanaka, K.; Chujo, Y. Polym. J. 2013, 45, 247-254.

41. Tanaka, K.; Jeon, J.-H.; Inafuku, K.; Chujo, Y. Bioorg. Med. Chem. 2012, 20, 915-919.

42. Tanaka, K.; Murakami, M.; Jeon, J.-H.; Chujo, Y. Org. Biomol. Chem. 2012, 10, 90-95.

43. Tanaka, K.; Ishiguro, F.; Chujo, Y. Polym. J. 2011, 43, 708-713.

44. Tanaka, K.; Ishiguro, F.; Chujo, Y. J. Am. Chem. Soc. 2010, 132, 17649-17651.

45. Tanaka, K.; Kitamura, N.; Naka, K.; Morita, M.; Inubushi, T.; Chujo, M.; Nagao, M.; Chujo, Y. Polym. J. 2009, 41, 287-292.

46. Asuncion, M. Z.; Ronchi, M.; Abu-Seir, H.; Laine, R.M. C. R. Chimie 2010, 13, $270-281$.

47 Tanaka, K.; Adachi, S.; Chujo, Y. J. Polym. Sci. Part A: Polym. Chem. 2009, 47, 5690-5697.

48. Tanaka, K.; Inafuku, K.; Chujo, Y. Chem. Commun. 2010, 46, 4378-4380.

49. Tanaka, K.; Okada, H.; Jeon, J.-H.; Inafuku, K.; Ohashi, W.; Chujo, Y. Bioorg. Med. Chem. 2013, 21, 2678-2681.

50. Kitamura, N.; Hiraoka, T.; Tanaka, K.; Chujo, Y. Bioorg. Med. Chem. 2012, 20, 4668-4674.

51. Inoue, H.; Watanabe, Y.; Masuno, A.; Kaneko, M.; Yu, J. Opt. Mater. 2011, 33, 1853-1857. 


\section{Tables and Figures}

Table 1. Thermal and optical properties of the polymer blends with or without $10 \mathrm{wt} \%$ of the polysulfidebridging POSS network

\begin{tabular}{ccccccc}
\hline & \multicolumn{2}{c}{ PMMA } & & \multicolumn{2}{c}{ PS } \\
\cline { 2 - 3 } \cline { 5 - 6 } \cline { 5 - 6 } POSS content & $0 \mathrm{wt} \%$ & $10 \mathrm{wt} \%$ & & $0 \mathrm{wt} \%$ & $10 \mathrm{wt} \%$ \\
\hline$T_{20 \mathrm{~d}}\left({ }^{\circ} \mathrm{C}\right)^{a}$ & 263 & 360 & & 388 & 358 \\
$T_{\mathrm{g}}\left({ }^{\circ} \mathrm{C}\right)$ & 68 & 64 & & 73 & 61 \\
$n_{\mathrm{D}}$ & 1.4931 & 1.4994 & & 1.5728 & 1.5791 \\
$\nu_{\mathrm{D}}$ & 60 & 59 & & 33 & 32 \\
\hline
\end{tabular}

${ }^{a}$ Determined from the decomposition temperatures with $20 \mathrm{wt} \%$ mass loss. 
Scheme 1. Synthesis of the polysulfide-bridging POSS network and the estimated chemical structure of the single POSS unit in the network ${ }^{a}$

$$
\begin{aligned}
& \mathrm{S}_{8} \stackrel{\mathrm{a}}{\longrightarrow} \mathrm{Na}_{2} \mathrm{~S}_{\mathrm{x}} \stackrel{\mathrm{b}}{\longrightarrow} \\
& X=\text { av. } 5
\end{aligned}
$$

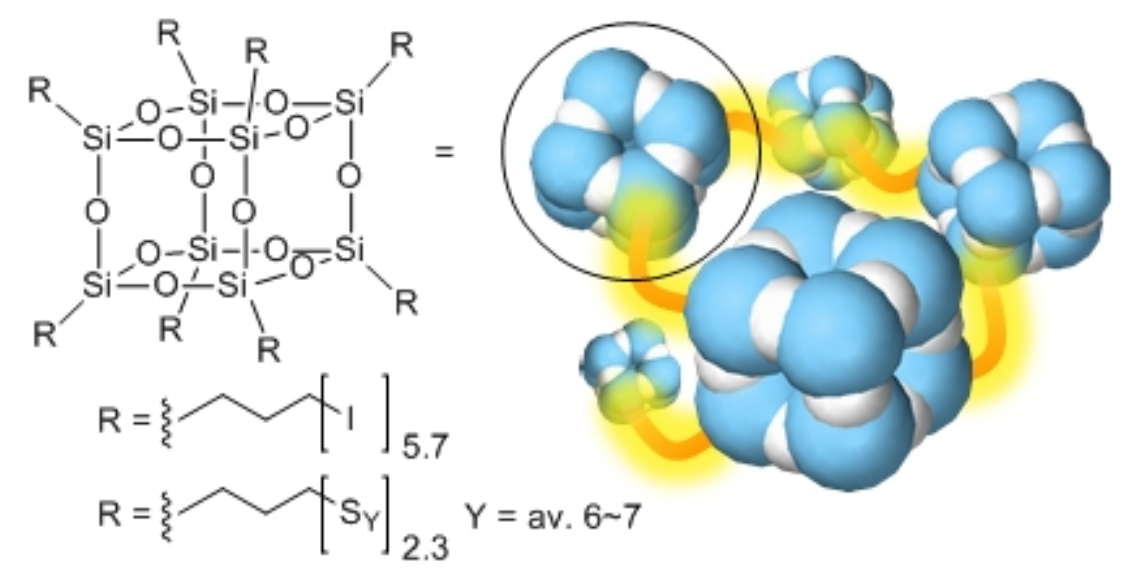

${ }^{a}$ Reagents and condition: (a) Na, THF, $70{ }^{\circ} \mathrm{C}, 3$ h; (b) octakis(3-iodopropyl)POSS, r.t., 30 min. 
a

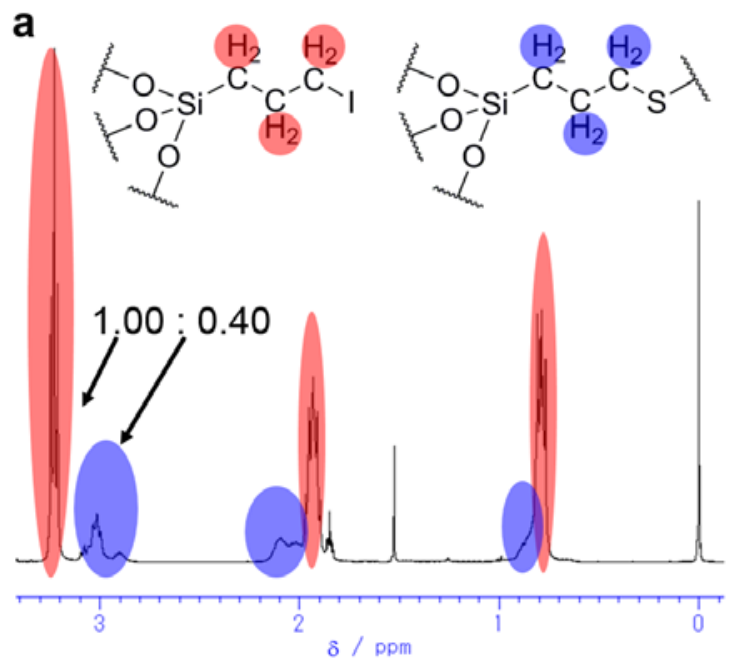

b

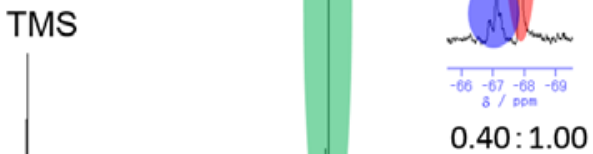

glass

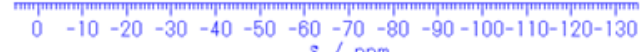

Figure 1. (a) ${ }^{1} \mathrm{H}$ and (b) ${ }^{29} \mathrm{Si}$ NMR spectra of the polysulfide-bridging POSS network in $\mathrm{CDCl}_{3}$. 


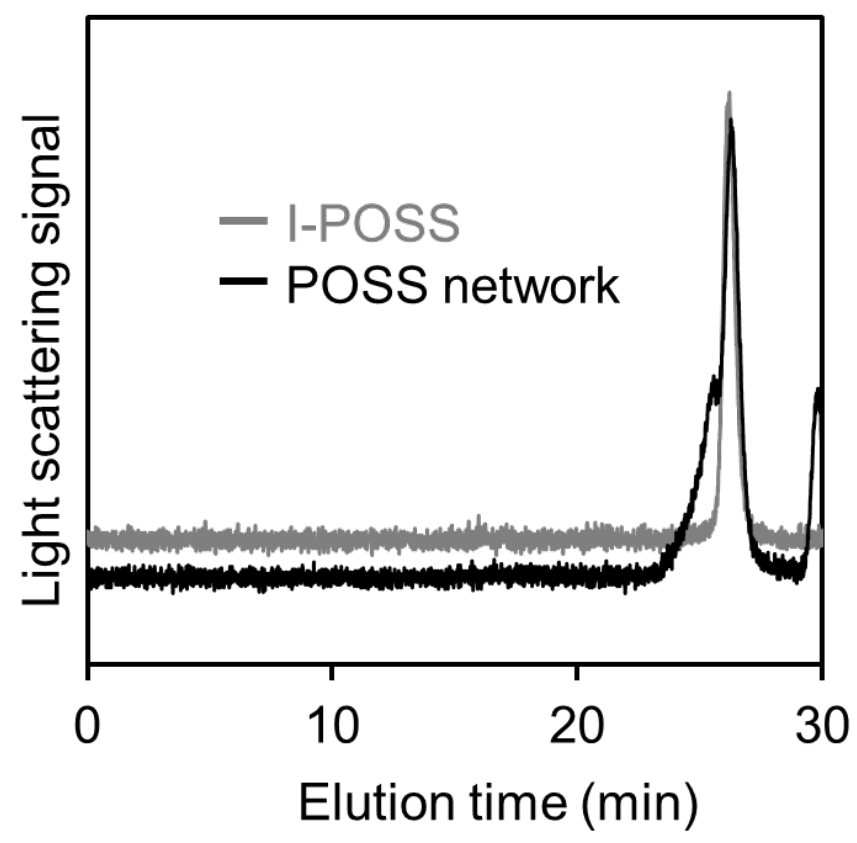

Figure 2. GPC-LALS profiles of octakis(3-iodopropyl)POSS (gray line) and the polysulfide-bridging POSS network (black line). 
PMMA

errectuvely with POSS derivatives than with polymers. ${ }^{11 a}$ Consequently, very porous materials with higher modulus, large surface area, and low thermal conductivity-were obtained. In this section, the ef ects of the POSS molecules on therma and mechanical properties of conventional polymeri arc explained.

V/e inve stiga ed the relationship between the enhancement of the physical properties and the chemical structures of the substitutio 1 groups in the POSS derivatives lis ed in Table $1 .^{12}$ Honogeneous polymer composites with polys:yrene (PS and poly(methy 1 methacrylate) (PMMA) were prepared with various concentrations of octa-subst tuted aliphatic and aromatic POSS. Differential scanning calorimetry (DSC) mei surements suggested tha: the POSis fillers could disperse in the polymer matices and nteract. with each polymer chain via strong hydrophobic interaction.

$0 w t \%$ t 5 wt $\%$ s $10 w t \%$ wt 15 wt 20

\section{PS}

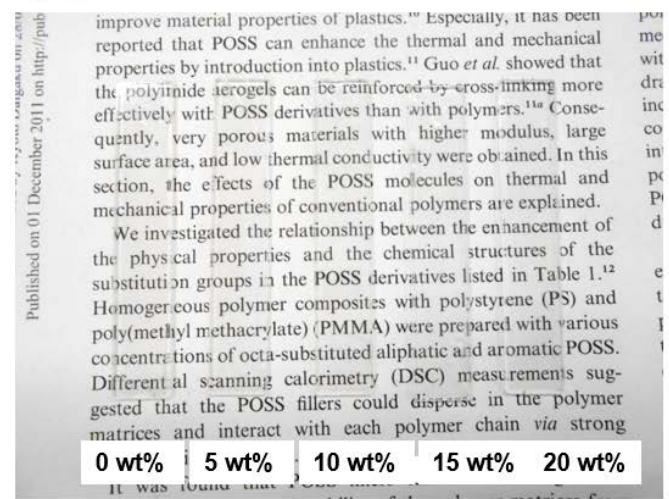

Figure 3. Appearances of the polymer blends with various amounts of the polysulfide-bridging POSS network. 

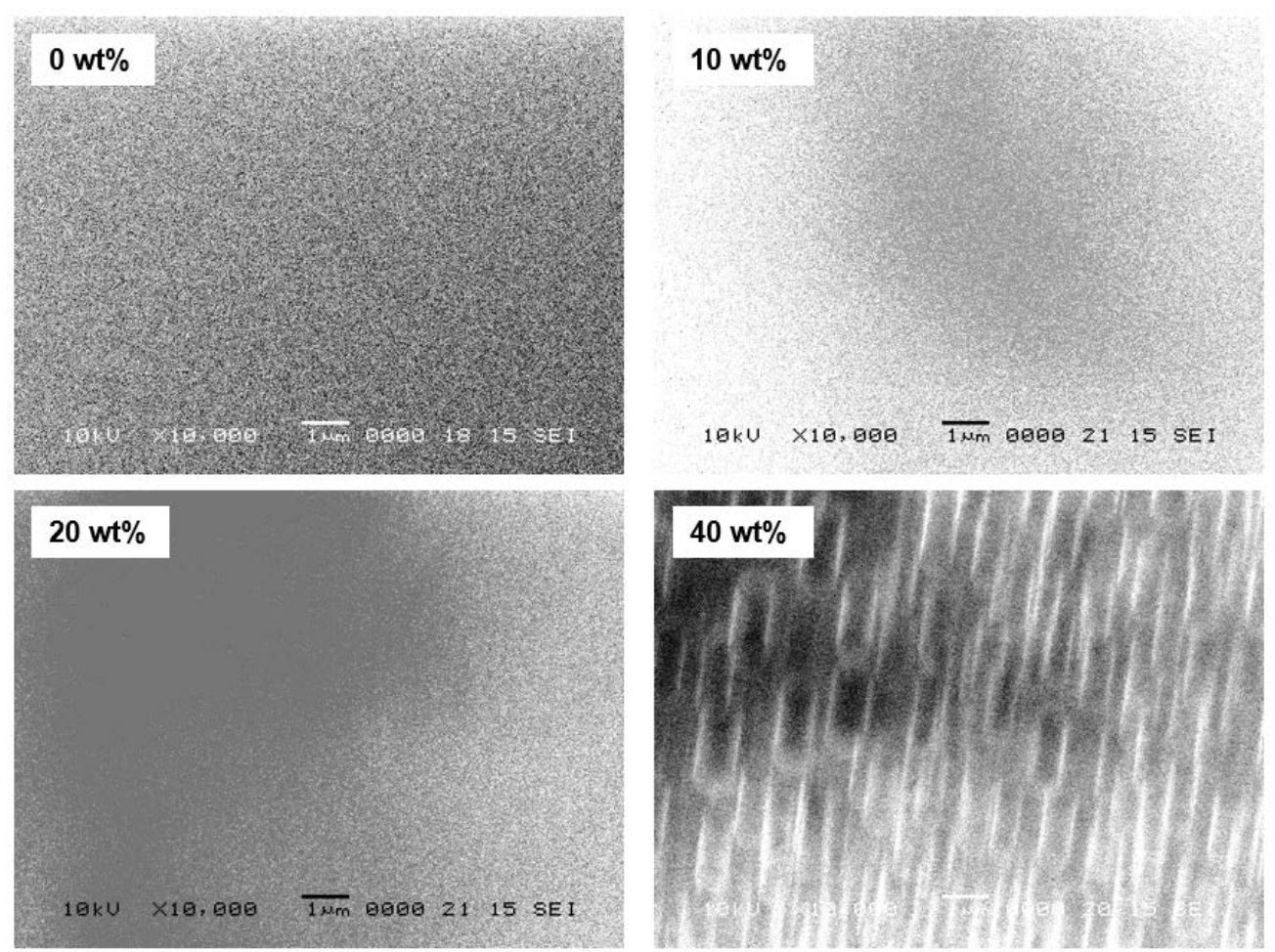

Figure 4. SEM observations of the PMMA blend films with various amounts of the polysulfidebridging POSS network. Scale bars represent $1 \mu \mathrm{m}$. 

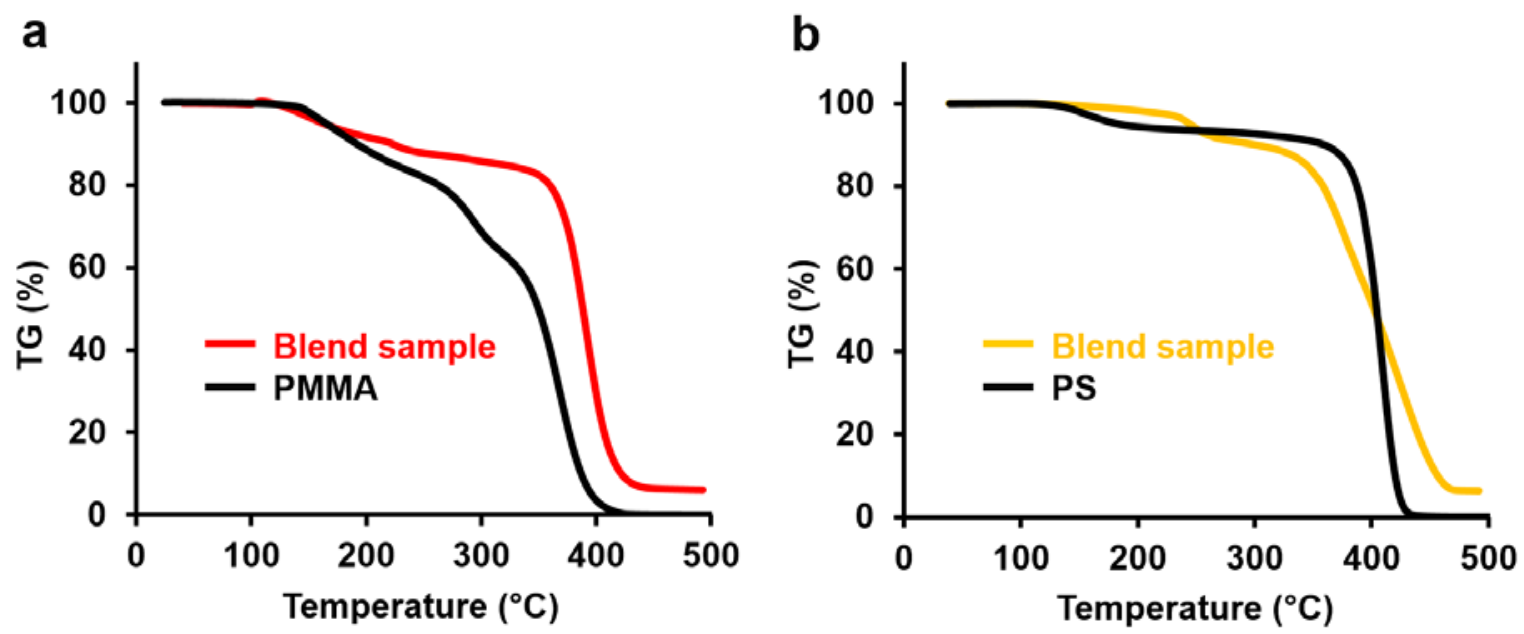

Figure 5. TGA thermograms of (a) PMMA and (b) PS blends containing $10 \mathrm{wt} \%$ of the polysulfidebridging POSS network with a heating rate of $10^{\circ} \mathrm{C} / \mathrm{min}$ under nitrogen atmosphere. 

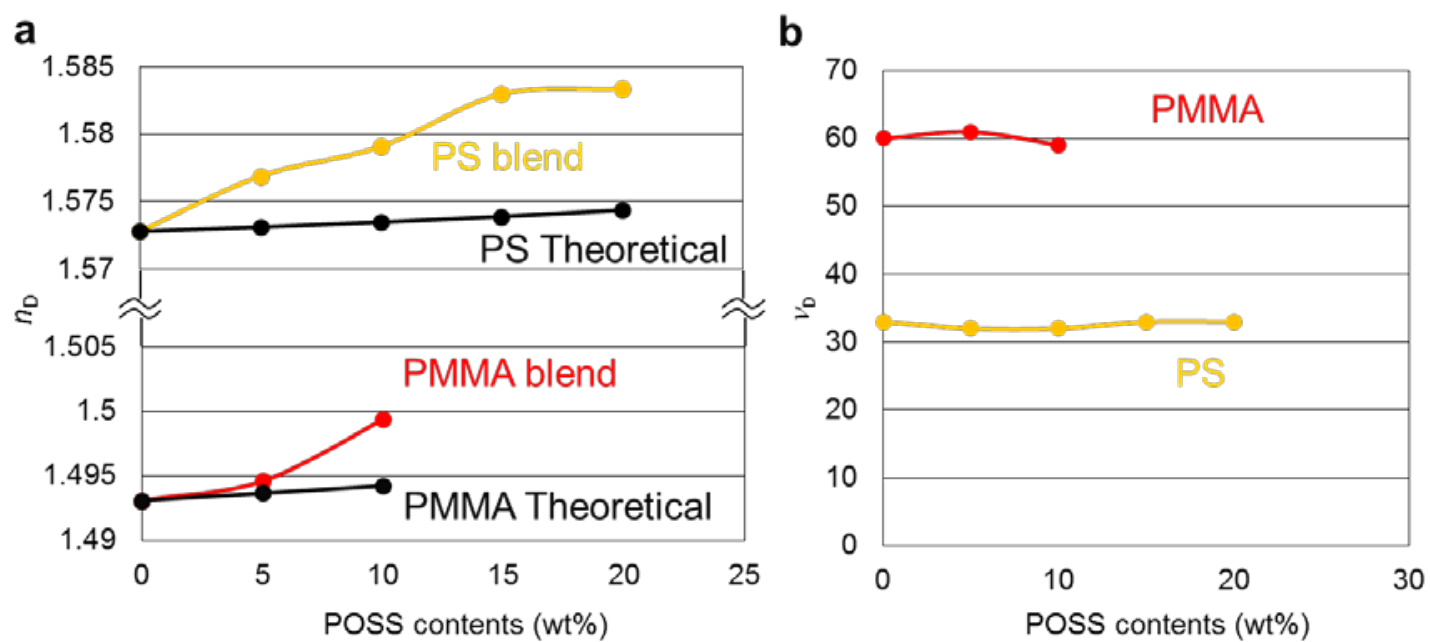

Figure 6. (a) Refractive indices $\left(n_{\mathrm{D}}\right)$ and (b) Abbe numbers $\left(v_{\mathrm{D}}\right)$ of the polymer blends with various amounts of the polysulfide-bridging POSS network. 